\title{
NOTE
}

\section{Qualitative patterns and processes in marine ecology: a conceptual programme}

\author{
R. H. Bradbury ${ }^{1}$, D. G. Green ${ }^{2}$ \& R. E. Reichelt ${ }^{1}$ \\ 1 Australian Institute of Marine Science, PMB 3, Townsville MSO, Queensland 4810, Australia \\ ${ }^{2}$ Department of Biogeography and Geomorphology, Australian National University, Canberra 2601, Australia
}

\begin{abstract}
Since ecosystem organization is a qualitative phenomenon, ecological pattern and process, its static and dynamic manifestations, are best captured as qualitative models. However, ecological methodology is overwhelmingly quantitative in orientation - a reflection not only of the transfer to ecology of the physical sciences paradigm, but also of the paucity of qualitative techniques. We argue that avenues now exist for the rapid exploitation of the qualitative approach. This holds the potential both of modelling ecological systems in terms of intrinsically relevant variables and of creating an independent ecological paradigm.
\end{abstract}

\section{THE PROBLEM}

\section{The nature of biological phenomena}

Pattern and process are dual concepts in biology: they are static and dynamic reflections of organization in biological systems. Biological patterns emerge through biological processes and biological processes are rich in patterns.

In this essay, we will explore the qualitative aspects of this duality in the context of marine ecology, since much biological organization and, hence, many biological patterns and processes are essentially qualitative in nature. We will seek to show that much significant pattern and process may be approached directly through appropriate qualitative technique, rather than indirectly through awkward quantitative mapping. This is not to reject completely the quantitative approach to biology but to suggest that it is a subset of a more general analytical approach. We will argue that the set of essential elements - a conceptual programme - of a qualitative approach to biological pattern and process now exists. We will support our argument by appeal to our recent qualitative studies on the structure and function of the complex, high diversity ecosystems of tropical coral reefs. Then we will suggest that while the elements of such a conceptual program exist - an expanding repertoire of tools for capturing qualitative data and identifying qualitative variables, as well as theories about the qualitative behaviour of biological systems - there is now an urgent need to integrate these elements into a coherent qualitative approach to pattern and process in marine ecology.

\section{Limitations of numerical methods}

Quantitative methods have been extremely valuable in biology in the sense that the biology we have today is, in large measure, a consequence of the quantitative approach. However, several general shortcomings limit their future utility in reaching a more comprehensive understanding of biological phenomena.

Firstly, they make tacit assumptions that are often not biologically valid. For example, linear regression is used almost universally in biology, even where wide departures from linearity, normality, and continuity occur. Virtually all multivariate methods assume orthogonality amongst independent variables, but the biological systems they deal with are usually rich in interactions (Levins \& Lewontin 1980).

Second, 'size' variables (that is, ones taking numerical values and in contrast to 'shape' variables expressing qualitative aspects) used in biology are often 'unnatural' and ignore vital features. It is often a challenge to devise numerical variables to represent patterns or processes such as animal behaviour or plant growth. By forcing data to become quantitative, biologists ignore important, sometimes critical, qualitative features of systems. Much information is lost if one describes an object's size but not its shape or appearance, yet that is exactly what is often done.

Consider, for example, the problem of ecological 
classification of a coral reef community. A quantitative approach (Loya 1972) measured the distribution and abundance of the various coral species in different parts of the reef. The pattern of the community structure (itself a qualitative result) was extracted from the high dimension ensemble of 'size' variables (the distribution and abundance measures) by the dimensionreducing techniques of numerical classification. However, this process did not recover all the significant pattern in the community. A qualitative approach, using the heuristic (or learning) algorithm CRL (Notley 1970), dealt with the same data as if it were a text from an unknown language (Bradbury \& Loya 1978). It generated 'shape' variables directly in the form of words (patterns of co-occurring corals) and analysed the behaviour of these variables. This analysis revealed a deep structure predicated on coherent levels of organization above the species. This structure was not recovered through conventional techniques employing 'size' variables.

The success of numerical methods in physical sciences is often viewed as a paradigm for how science should be done. Certain quantitative concepts (e.g. information, diversity) have thus become fashionable amongst biologists. The prevalent attitude is that only numerical data is 'hard' (reliable), yet often only the so-called 'soft' data conveys the true nature of a biological process (Mayr 1982). A consequence is that much research has concentrated on quantitative questions sometimes trivial ones - while many qualitative, organizational questions have received little or no attention. For example, Hogeweg \& Hesper (1984) have shown that the traditional quantitative aspects of population distribution and abundance and their simple correlation with environmental parameters are not nearly as informative about bumble bee colony behaviour as are non-traditional, qualitative attributes of local bee-to-bee interactions.

\section{The need}

Interest in the automated acquisition and analysis of qualitative data has grown explosively amongst biologists in the past decade. Research into pattern recognition and artificial intelligence is rapidly yielding a wide range of such systems for use in biology (e.g. Hogeweg \& Hesper 1974, Siler \& Lindberg 1974, Pankhurst 1975, Kumur 1977, Buchanan \& Feigenbaum 1978, Stefik 1978, Oliver et al. 1979, Wheeler \& Misra 1980, Vanderheydt et al. 1981).

Given this interest and the limitations of numerical methods 3 needs can be identified that are common to many areas of biology:

(1) There is a need for techniques that allow qualita- tive data to be recorded systematically, concisely, and with known reliability - for example, that the qualitative attribute colour equals (blue, green, other) and not that the quantitative attribute colour equals, say $(450 \mathrm{~nm})$.

(2) Given that the first need can be met, there is a need to identify key 'shape' variables that reflect patterns or processes of biological significance - for example, these could vary from the postulated 'self-organizing' property of ecosystems (Ulanowicz 1979b) to the properties of food webs when considered as formal graphs (Cohen 1978).

(3) Given that the first 2 needs can be met, there is a need to develop specific and general theories about qualitative features of biological systems - for example, extending and applying the mathematical theories of topology into biology (Winfree 1980).

Together these needs form the skeleton of a conceptual programme for a qualitative ecology of marine systems. We will now develop each of them in turn.

\section{A PROGRAMME}

\section{Design the mathematics to suit}

There is no comprehensive mathematics of 'shape' variables at present. However, the potential for such a mathematics now exists using the theory of formal languages. Stimulated by research into automata theory, pattern recognition, and artificial intelligence, much work has gone into the development of formal languages and associated ideas (e.g. Hopcroft \& Ullman 1969, Zadeh 1973, Fu 1974). Formal languages are systems for producing descriptions by manipulating symbols according to well-defined rules. Strictly speaking, a formal language is defined by a grammar consisting of an alphabet (a finite set of symbols used by the language), a set of starting words (arrangements of symbols from the alphabet) and a set of production rules (a finite set of rules that specify how to create new words from old words). A particular formal language consists of all words that can be derived by applying a finite sequence of production rules to the starting words.

It should be emphasized that the theory of formal languages is not divorced from conventional mathematics. On the contrary, all the symbolism of conventional (i.e. numerical) mathematics comprises one particular formal language - the language of arithmetic (Whitehead \& Russell 1910-1913). All computer languages are formal languages, as too are all diagrammatic methods for representing data. There are 4 reasons why formal languages can serve as a basis for a 
mathematics of pattern. (1) Any qualitative pattern can be represented by an appropriate formal language. For example, a formal language approach immediately establishes that the differences or similarities between a square and a triangle depend as much upon the observer (for the definition of symbols, rules and words) as they do upon the objects observed. (2) Formal language can be designed to reflect what are considered to be truly important biological patterns and processes in a particular situation. (3) Computers now make it possible to manipulate formal language descriptions quickly and easily. (4) Even if simulations are discounted, the number of applications of formal languages to biology is growing rapidly (e.g. Newell 1970, Herman \& Rozenberg 1975, Westerman 1977. Bradbury \& Loya 1978, Haeffner 1978, Schmidt et al. 1978, Haken 1979, Papentin 1980).

The formal language approach has 2 additional benefits. (1) The relation between a mathematics of pattern and numerical mathematics becomes clear. (2) Problems that are transcomputable in numerical terms (and hence unanalysable with numerical mathematics, see Bremermann 1977) may be tackled with qualitative heuristic procedures which are formal languages (Wimsatt 1982).

Whilst a large repertoire of concepts and techniques is growing up around formal languages and their applications, the discipline has as yet no all-embracing theoretical basis. Thinking of shape variables as a generalization of size variables suggests ways in which analogues or generalizations of important theoretical concepts associated with size variables might be found. For instance:

(1) The concept of measurement can readily be generalized to include scales in which the values are descriptions rather than numbers. Measurement is not just getting numbers but rather a formal procedure for recording observations. Numerical measurement maps observations onto the real line, syntactic measurement maps observations onto a different ensemble. (2) Programmes that manipulate descriptions generalize the concept of algebraically defined functions. (3) Tools such as 'expert' or 'learning' systems (e.g. Ivakhnenko 1971) and 'evolutionary programming' (e.g. Fogel et al. 1966) are analogous to regression with numerical variables.

Coral reef ecosystems have provided a fertile testing ground for such tools, allowing incremental improvements in technique as well as insights into the nature of these complex systems. Bradbury \& Loya (1978), in their study of the spatial patterns of the coral reef benthos using the heuristic (or learning) algorithm CRL, were able to recover patterns which demonstrated that the community was well-structured, and that this structure was intrinsically hierarchical with coherent levels of organization above the species. More recently, Green et al. (1983) used the GMDH algorithm of Ivakhnenko (1971), a heuristic analogue of multiple regression, to establish the patterns of predictability in a coral reef community. These patterns were strong, and independent of consistent patterns of dissimilarity within and between the reefs derived from other numerical studies. Reichelt et al. (1983), in another recent study, used graph theoretic techniques to establish strong structural patterns in coral reef communities independent of the structures derived from numerical studies. All 3 of these essentially qualitative studies have used techniques, novel to coral reef ecology at least, to capture new patterns in the system, unrevealed by conventional quantitative studies, and which point to hitherto unsuspected deep structure.

\section{Identify key concepts and variables}

The formal language approach suggests that, if the physical sciences paradigm has any value, then it will be mapped into biology at a much higher and more abstract level than the present wholesale mapping into biology of physical concepts. The mapping will be between formal languages rather than within the one constraining arithmetic language of physical science. Thus there should be, and we should seek, concepts comparable to mass, force, change and time that are relevant to biology (Prigogine 1980). Perhaps Ulanowicz's (1979a, b) 'self-organizing' property of ecosystems is a step in this direction.

One way to approach this question is to try to generalize existing ideas. We can talk about the quality of information, for example. All biological processes involve information transfer. Measuring the types of information transferred (and how it is transferred) may prove more useful than measuring how much information is transferred (Ryan 1980). Information theory was developed in the context of electronic communications (Shannon \& Weaver 1949) and, because of the duality between signal noise and system entropy, it has become closely associated with thermodynamics (Prigogine 1980). A major shortcoming encountered in applying these ideas to biological systems is that the crucial role of organization (and the consequent chunking of information, Ryan 1980) is ignored. Papentin (1980) showed that this deficiency can be overcome by making the crucial distinction between the organized and random components of biological complexity. 'Organized complexity' is measured by finding the length of a minimal grammar that describes a pattern's underlying organization. 'Random complexity' consists of all non-regular features in 
a pattern. Papentin argues that only random complexity is constrained by the Second Law of Thermodynamics and that major trends in the course of evolution have been for total complexity to increase and for organized complexity 'to emerge from, and at the expense of, random complexity'.

'Pattern' and 'process' are themselves universal ideas in biology constantly re-emerging at all levels from genetics (Waddington 1957) to ecology (Williams 1964, Miller 1967, Southwood 1982). There is probably much to gain from developing standard languages and automata for dealing with particular sorts of patterns and processes. One advantage of standard languages is that interpretive methodologies can be developed. For example, remote sensing raises the possibility of monitoring patterns and processes over large areas of the oceans. Because remote sensing data can be considered as complex measurements of marine ecosystem structure expressed in a formal language, and because interpretation (or enhancement) of the data can be structured syntactically (Fu 1974), the possibility exists that these data may be more profitably explored through a qualitative approach than the currently used numerical methods.

A more subtle reason for developing standard languages is that treating different phenomena in similar ways has led to some of the most profound insights in science. One concept with widespread biological relevance is that of 'cellular automata' (Wolfram 1984). A 'cellular automaton' is any structured population of cells, all of which behave as identically programmed automata. Thus developmental models (Herman \& Rozenberg 1975) are cellular automata in which the cell population is allowed to grow. On a larger scale, a coral reef simulation may be defined by using a rectangular array of cells to represent locations in space, and then assigning states to the cells corresponding to different coral types, plus substrate (Reichelt et al. 1985).

We have already begun trying to find expression for these ideas - of mapping the physical sciences paradigm into biology and of generalizing concepts of biological pattern and process - in the domain of coral reef ecology. Recent theoretical studies (Bradbury et al. 1983, 1984) have argued that, for complex ecosystems such as coral reefs, there is no simple mapping of the identity between predictive and explanatory models which is so well established under the physical sciences paradigm. They have also argued against the 'true causes' and programmatic reductionism implicit in many attempts to undertake the mapping. Empirical studies have confirmed the power of treating prediction and explanation as separate concepts for understanding coral reef ecosystems (Green et al. 1983, Reichelt et al. 1983) as described earlier.

\section{Develop qualitative theories}

Given that we can design the mathematical tools to record and manipulate qualitative patterns and processes, and that we can identify the key concepts and variables emerging from such a qualitative approach, what should our qualitative theories look like? Can we be confident that robust theories derived from a qualitative approach will emerge - whether they are theories specific to the organization of marine ecosystems or apply to all ecosystems or even generally to all biological systems?

There is cause for optimism. The studies by Bradbury \& Loya (1978) described above used qualitative techniques to establish a deep structure for coral reef communities while conventional quantitative techniques could only reveal the epiphenomena of systematic environmental zonation (Loya 1972). The qualitative theory of ecosystem structure developed from these studies has significance not only for coral reef ecosystems, but more generally for other complex ecosystems (Bradbury 1977).

In another study, Bradbury et al. (1985) used topological techniques to describe the qualitative dynamics of the interaction between the crown-of-thorns starfish Acanthaster planci and the coral community. The analysis not only leads to a useful and parsimonious description of those dynamics, but also extends the classical quantitative ecological theory of population limit cycles into a hitherto intractable domain.

In fact, qualitative theories of ecosystem structure are more prevalent than many scientists would probably admit. Partridge \& Lopez (1984) have pointed out that simulation models are effectively qualitative theories about the systems that they represent, since they embody sets of assumptions about the modelled systems. In our work (e.g. Green et al. 1984, Reichelt et al. 1985) we have tried to take their argument one step further. Rather than embody particular assumptions, our simulation programmes model ecosystem processes in such a way that they embody a 'biological language' within which simple hypotheses and assumptions may be defined. Thus in the coral reef simulation described above, assumptions about competition can be represented as a set of replacement formulae such as Branching Coral $\rightarrow$ Massive Coral.

\section{CONCLUSION}

The orientation of modern marine ecology, like so much of biology, is overwhelmingly quantitative. The methodology, the concepts and the theory all bear witness to things quantitative, despite a recognition of the qualitative nature of ecological systems. We argue 
against this world-view: that since ecological organization is a qualitative phenomenon, ecological pattern and process, its static and dynamic manifestations, are best captured as qualitative models.

We recognize that the awkward quantitative mappings of fundamentally qualitative ecological phenomena, so characteristic of much of recent marine ecology, reflect not only the simplistic transfer to ecology of the physical sciences paradigm, but also the paucity of qualitative techniques. However, we argue that avenues now exist - in terms of tools, concepts and theories - for the rapid exploitation of the qualitative approach in marine ecology. This holds the potential both of modelling our marine ecosystems in terms of intrinsically relevant variables and of creating an independent ecological paradigm. The urgent task is now to fully integrate these approaches into a coherent programme.

Acknowledgements. This is AIMS Contribution Number 314.

\section{LITERATURE CITED}

Bradbury, R. H. (1977). Independent lies and holistic truths: towards a theory of coral reef communities as complex systems. Proc. Third Int. Coral Reef Symp. 1:1-7

Bradbury, R. H., Hammond, L. S., Moran, P. J., Reichelt, R. E. (1985). Coral reef communities and the crown-of-thorns starfish: evidence for qualitatively stable cycles. J. theor Biol. 113: 69-80

Bradbury, R. H., Hammond, L. S., Reichelt, R. E., Young, P. C. (1983). Prediction versus explanation in environmental impact assessment. Search 14: 323-325

Bradbury, R. H., Hammond, L. S., Reichelt, R. E., Young, P. C. (1984). Prediction and explanation as a dialectical pair in ecology. Search 15: 220-222

Bradbury, R. H., Loya, Y. (1978). A heuristic analysis of spatial pattern of hermatypic corals at Eilat Red Sea. Am. Nat. 112: 493-507

Bremermann, H. J. (1977). Complexity and transcomputability. In: Bloggs, B. (ed.) The encyclopaedia of ignorance. Pergamon Press, Oxford, p. 167-174

Buchanan, B. G., Feigenbaum, E. A. (1978). Dendral and Meta-Dendral: their applications dimension. Artificial Intelligence 11: 5-24

Cohen, J. E. (1978). Food webs and niche space. Princeton University Press, New Jersey

Fogel, L. J., Owens, A. J., Walsh, M. J. (1966). Artificial intelligence through simulated evolution. John Wiley and Sons, New York

Fu, K. S. (1974). Syntactic methods in pattern recognition. Academic Press, New York

Green, D. G., Bradbury, R. H., Reichelt, R. E. (1983). Explanation, prediction and control in coral reef ecosystems II. Models for prediction. In: Baker, J. T. et al. (ed.) Proceedings of Inaugural Great Barrier Reef Conference. James Cook University Press, Townsville, p. 213-215

Green, D. G., House, A. P. N., House, S. M. (1985). Simulating spatial pattern in forest ecosystems. Mathematics and computers in simulation (in press)
Haeffner, J. W. (1978). Ecosystem assembly grammars: generative capacity and empirical adequacy. J. theor. Biol. 73: 293-318

Haken, H. (ed.) (1979). Pattern formation by dynamic systems and pattern recognition. Springer-Verlag, Berlin

Herman, G. T., Rozenberg, G. (1975). Development systems and languages. North-Holland, Amsterdam

Hogeweg, P., Hesper, B. (1974). A model study on biomorphological description. Pattern Recognition 6: 165-179

Hogeweg, P., Hesper, B. (1984). The ontogeny of the interaction structure in bumble bee colonies: A MIRROR model. Behav. Ecol. Sociobiol. 12: 271-283

Hopcroft, J. E., Ullman, J. D. (1969). Formal languages and their relation to automata. Addison-Wesley, Reading

Ivakhnenko, A. G. (1971). Polynomial theory of complex systems. IEEE Transactions on Systems, Man and Cybernetics 1: $364-378$

Kumur, A. (1977). A real-time system for pattern recognition of human sleep stages by fuzzy system analysis. Pattern Recognition 9: 43-46

Levins, R., Lewontin, R. (1980). Dialectics and reductionism in ecology. Synthese 43: 47-78

Loya, Y. (1972). Community structure and species diversity of hermatypic corals at Eilat, Red Sea. Mar. Biol. 13: 100-123

Mayr, E. (1982). The growth of biological thought. Belknap Press, Massachusetts

Miller, R. S. (1967). Pattern and process in competition. Adv ecol. Res. 4: 1-74

Newell, A. (1970). Remarks on the relationship between artificial intelligence and cognitive psychology. In: Banerji R., Mesarovic, M. D. (ed.) Theoretical approaches to nonnumerical problem solving. Springer-Verlag, New York, p. $363-400$

Notley, M. G. (1970). The cumulative recurrence library Comput. J. 13: 14-19

Oliver, L. H., Poulsen, R. S., Toussaint, G. T., Louis, L. (1979) Classifications of atypical cells in the automatic cytoscreening for cervical cancer. Pattern Recognition 11: 205-212

Pankhurst, R. J. (ed.) (1975). Biological identification with computers. Academic Press, London

Papentin, F. (1980). On order and complexity. I. General considerations. J. theor. Biol. 87: 421-456

Partridge, D., Lopez, P. D. (1984). Computer programs as theories in biology. J. theor. Biol. 108: 539-564

Prigogine, I. (1980). From being to becoming. Freeman, San Francisco

Reichelt, R. E., Green, D. G., Bradbury, R. H. (1983). Explanation, prediction and control in coral reef ecosystems $\mathrm{I}$. Models for explanation. In: Baker, J. T. et al. (ed.) Proceedings of Inaugural Great Barrier Reef Conference. James Cook University Press, Townsville, p. 231-235

Reichelt, R. E., Green, D. G., Bradbury, R. H. (1985). Discrete simulation of cyclone effects on the spatial patterns and community structure of a coral reef. Proc. Fifth Int. Coral Reef Congress, Tahiti (in press)

Ryan, J. P. (1980). Information-entropy interfaces and different levels of biological organization. J. theor. Biol. 84: $31-48$

Schmidt, C. F., Sridharan, N. S., Goodson, J. L. (1978). The plan recognition problem: an interaction of psychology and artificial intelligence. Artificial Intelligence 11:45-84

Shannon, C. E., Weaver, W. (1949). The mathematical theory of communication. Univ. Illinois Press, Chicago

Siler, W., Lindberg, D. A. (ed.) (1974). Computers in life science research. Plenum Press, New York

Southwood, T. R. E. (1982). Ecology - A mixture of pattern and 
probabilism. In: Saarinen, E. (ed.) Conceptual issues in ecology. Reidel, Dordrecht, p. 203-214

Stefik, M. (1978). Inferring DNA structures from segmentation data. Artificial Intelligence 11: 85-114

Ulanowicz, R. E. (1979a). Prediction, chaos, and ecological perspective. In: Halfron, E. (ed.) Theoretical systems ecology, Academic Press, New York, p. 107-117

Ulanowicz, R. E. (1979b). Complexity, stability and selforganization in natural communities. Oecologia (Berl.) 43 295-298

Vanderheydt, L., Dom, F., Oosterlink, A., van der Berghe, H. (1981). Two-dimensional shape decomposition using fuzzy subset theory applied to automated chromosome analysis. Pattern Recognition 13: 147-158

Waddington, C. H. (1957). The strategy of the genes. George Allen and Unwin, London

Westman, R. S. (1977). Environmental languages and the functional basis of behaviour. In: Hazlett, B. A. (ed.) Quantitative methods in the study of animal behaviour. Academic Press, New York, p. 145-201

Wheeler, S. G., Misra, P. N. (1980). Crop classification with
Landsat multispectral scanner data II. Pattern Recognition 12: 219-228

Whitehead, A. N. Russell, B. A. W. (1910-1913). Principia mathematica. Vol. I, 1910; Vol. 2, 1912; Vol. 3, 1913. Cambridge University Press, Cambridge

Williams, C. B. (1964). Patterns in the balance of nature and related problems in quantitative biology. Academic Press, New York

Wimsatt, W C. (1982). Reductionistic research strategies and their biases in the units of selection controversy. In: Saarinen, E. (ed.) Conceptual issues in ecology. Reidel, Dordrecht, p. 155-201

Winfree, A. T. (1980). The geometry of biological time. Springer-Verlag, New York

Wolfram, S. (1984). Cellular automata as models of complexity. Nature, Lond. 311: 419-424

Zadeh, L. A. (1973). Outline of a new approach to the analysis of complex systems and decision processes. IEEE Transactions on Systems, Man and Cybernetics 3: 28-44

Accepted for printing on November 12, 1985 